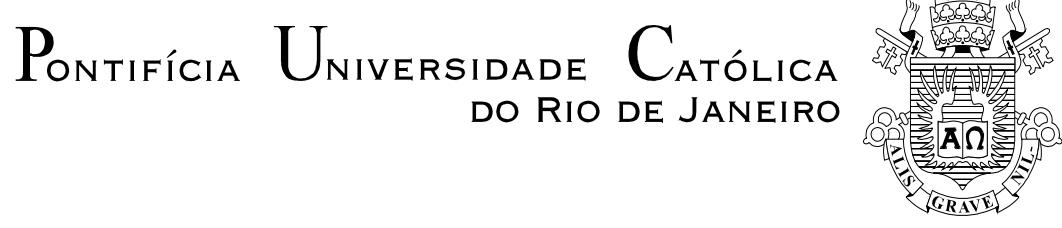

Daniel Armando Arias Gómez

Identificação das Barras Críticas do Ponto de Vista de Estabilidade de Tensão

Dissertação de Mestrado

Dissertação apresentada como requisito parcial para obtenção do grau de Mestre pelo Programa de Pósgraduação em Engenharia Elétrica do Departamento de Engenharia Elétrica da PUC-Rio.

Orientador: Prof. Ricardo Bernardo Prada

Rio de Janeiro

Julho 2015 


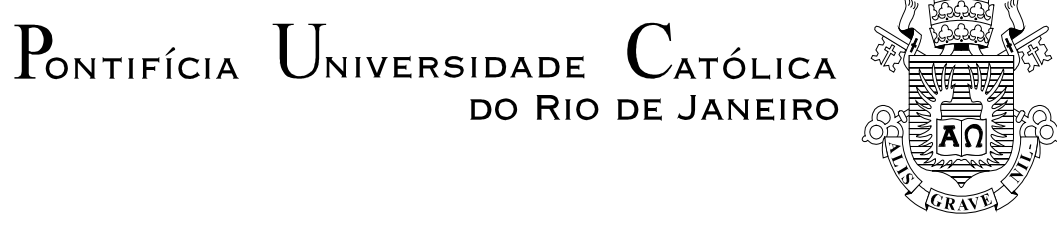

Daniel Armando Arias Gómez

\title{
Identificação das Barras Críticas do Ponto de Vista de Estabilidade de Tensão
}

\begin{abstract}
Dissertação apresentada como requisito parcial para obtenção do grau de Mestre pelo Programa de PósGraduação em Engenharia Elétrica do Departamento de Engenharia Elétrica do Centro Técnico Científico da PUC-Rio. Aprovada pela Comissão Examinadora abaixo assinada.
\end{abstract}

Prof. Ricardo Bernardo Prada

Orientador

Departamento de Engenharia Elétrica - PUC-Rio

Prof. Luiz Claudio de Araujo Ferreira

ONS

Prof. Jorge Luiz de Araujo Jardim

HPPA

Prof. José Eugenio Leal

Coordenador Setorial do Centro

Técnico Científico - PUC-Rio

Rio de Janeiro, 16 de Julho de 2015 
Todos os direitos reservados. É proibida a reprodução total ou parcial do trabalho sem autorização da universidade, da autora e do orientador.

Daniel Armando Arias Gómez

Graduou-se em Engenharia Elétrica pela Universidad Nacional da Colombia - Manizales - Colombia em 2013.

Ficha Catalográfica

Arias Gómez, Daniel

Identificação das Barras Críticas do Ponto de Vista de Estabilidade de Tensão / Daniel Armando Arias Gómez; orientador: Ricardo Bernardo Prada - 2015.

v., 188 f.: il. (color.); $30 \mathrm{~cm}$

Dissertação (mestrado)-Pontifícia Universidade Católica do Rio de Janeiro, Departamento de Engenharia Elétrica, 2015.

Inclui referências bibliofráficas

1. Engenharia Elétrica - Teses. 2. Estabilidade de Tensão. 3. Colapso de Tensão. 4. Índices de Avaliação de Estabilidade de Tensão. 5. Ponto de Máximo Carregamento.

I. Prada, Ricardo Bernardo. II. Pontifícia Universidade Católica do Rio de Janeiro. Departamento de Engenharia Elétrica. III. Título

CDD: 621.3 


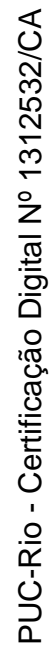

Para Armando e Fernanda, meus pais, pelo amor, apoio e confiança. 


\section{Agradecimentos}

Agradeço a Deus pela vida e pelas oportunidades oferecidas.

Agradeço a minha família, por todo amor, dedicação, paciência, apoio e confiança que estiveram presentes desde sempre.

Ao meu orientador Ricardo Bernardo Prada pela dedicação, paciência e orientação nas diferentes etapas do desenvolvimento nesta dissertação.

A CAPES e à PUC-Rio, pelos auxílios concedidos, sem os quais este trabalho não poderia ter sido realizado.

A meus amigos e colegas do laboratório de sistemas de energia da PUC-Rio, em especial a Fernando de Assis, José Filho e Maxwell Vargas.

Aos professores e funcionários Departamento de Engenharia Elétrica da PUC-Rio, pela qualidade do ensino e excelente infraestrutura, essenciais para a execução do curso de mestrado.

A meus grandes amigos Juan Simon Obando e Cesar Lopez, por terem sido meus parceiros nesta aventura. 


\section{Resumo}

Arias Gómez, Daniel Armando; Prada, Ricardo Bernardo. Identificação das Barras Críticas do Ponto de Vista de Estabilidade de Tensão. Rio de Janeiro, 2015. 188p. Dissertação de Mestrado - Departamento de Engenharia Elétrica, Pontifícia Universidade Católica do Rio de Janeiro.

O surgimento do fenômeno de estabilidade de tensão na operação dos sistemas elétricos motivou centenas de pesquisadores a desenvolverem métodos que permitam detectar a proximidade à instabilidade de tensão, com eficiência computacional, e tomar medidas acertadas para garantir a operação confiável e segura. Neste trabalho são utilizados e comparados diferentes métodos associados à análise de estabilidade de tensão. Para testes, foram utilizados vários sistemas sob diferentes níveis de carregamento, desde carga leve até o máximo carregamento, este obtido a partir do algoritmo de fluxo de potência continuado. $\mathrm{O}$ objetivo é localizar as barras críticas, isto é, aquelas onde não se pode aumentar a injeção de potência e que impedem o crescimento da carga do resto do sistema.

\section{Palavras-chave}

Estabilidade de tensão; margem de potência; colapso de tensão; ponto de máximo carregamento; carga leve; fluxo de potência continuado. 


\section{Abstract}

Arias Gómez, Daniel Armando; Prada, Ricardo Bernardo (Advisor). Identification of Critical Buses from the Voltage Stability Viewpoint. Rio de Janeiro, 2015. 188p. MSc. Dissertation - Departamento de Engenharia Elétrica, Pontifícia Universidade Católica do Rio de Janeiro.

The rise of voltage stability phenomenon in the operation of the electrical systems motivated hundreds of researchers to develop methods that allow them to detect the proximity to voltage instability, with computational efficiency, and take the right steps to ensure reliable and safe operation. This work used and compared different methods associated with the voltage stability analysis. For the tests were used various systems under different levels of loading, from light load to maximum load, this point obtained from the continuous power flow algorithm. The goal is to find the critical buses, that is, those at which the power injection cannot be increased and that avoid the growth of load in the rest of the system.

\section{Keywords}

Voltage stability; power range; voltage collapse; maximum loading point; light load; continuous power flow. 


\section{Sumário}

$\begin{array}{ll}\text { 1. Introdução } & 18\end{array}$

$\begin{array}{lr}\text { 1.1. Considerações Gerais } & 18\end{array}$

$\begin{array}{ll}\text { 1.2. Objetivos } & 19\end{array}$

$\begin{array}{lr}\text { 1.3. Estrutura da Dissertação } & 19\end{array}$

2. Diretrizes e Critérios de Segurança Relativos à Estabilidade de Tensão 21

2.1. Modelagens de Carga nas Análises Estática e Dinâmica 23

2.2. Método e Critérios Estabelecidos para Estudos de

Estabilidade de Tensão no Sistema Interligado Brasileiro [2]. 29

2.2.1. Estabelecimento do Método para Estudos de Estabilidade de Tensão.

3. Fluxo de Potência e Fluxo de Potência Continuado 33

3.1. Fluxo de Potência [3] [4] 33

$\begin{array}{ll}\text { 3.1.1. Introdução } & 33\end{array}$

3.1.2. Modelagem de Linhas e Transformadores 34

3.1.3. O problema dos Fluxos de Potência 40

3.1.4. Expressões Gerais dos Fluxos 43

3.1.5. Formulação Matricial $\quad 43$

3.1.6. Método de Newton-Raphson para Solução dos Fluxos de Potência 46

$\begin{array}{ll}\text { 3.1.7. Sumário da Seção } & 50\end{array}$

3.2. Fluxo de Potência Continuado 51

3.1.1. Princípio Básico [1] 51

3.1.2. Formulação Matemática 52

3.1.3. Passo Preditor 53

3.1.4. Passo Corretor 55

3.1.5. Seleção do Parâmetro de Continuação 56 
4. O Fenômeno da Estabilidade de Tensão [6] 57

$\begin{array}{ll}\text { 4.1. Introdução } & 57\end{array}$

4.2. Caracterização do Fenômeno de Estabilidade de Tensão 57

4.3. Impedância de Carga no Máximo Carregamento 65

$\begin{array}{ll}\text { 4.4. Limite de Estabilidade de Tensão } & 67\end{array}$

4.5. Capacitor em Paralelo na Barra de Carga 70

4.6. Sumário do Capítulo $\quad 75$

5. Índices de Avaliação da Estabilidade de Tensão 77

$\begin{array}{ll}\text { 5.1. Introdução } & 77\end{array}$

5.2. Método da Matriz D' 77

5.2.1. Módulo do Determinante da Matriz D' 79

5.2.2. Sinal do Determinante da Matriz D' 83

5.2.3. Interpretação dos Índices 85

5.2.4. Índices de Avaliação de Estabilidade de Tensão em
Barras Controladoras e de Tensão Controlada

5.3. Vetor Tangente [9] 96

5.4. Índice L [12] 98

5.4.1. Índice L para Sistemas Multi-nó 101

5.5. Análise de Autovalores e Autovetores 103

$\begin{array}{ll}\text { 6. Procedimentos e Resultados } & 107\end{array}$

$\begin{array}{lr}\text { 6.1. Sistema de } 6 \text { Barras } & 108\end{array}$

$\begin{array}{lr}\text { 6.2. Sistema de } 14 \text { Barras } & 115\end{array}$

6.3. Sistema de 30 Barras 122

$\begin{array}{lr}\text { 6.4. Sistema de } 39 \text { Barras } & 127\end{array}$

$\begin{array}{lr}\text { 6.5. Sistema de } 57 \text { Barras } & 134\end{array}$

6.6. Sistema de 118 Barras 143 
6.7. Testes com Limites de Geração no Sistema de 118 Barras 150

6.8. Resumo dos Testes 153

$\begin{array}{lr}\text { 7. Conclusões e Trabalhos Futuros } & 158\end{array}$

$\begin{array}{lr}\text { 7.1. Conclusões } & 158\end{array}$

$\begin{array}{lr}\text { 7.2. Trabalhos Futuros } & 160\end{array}$

8. Referências Bibliográficas 162

A Dados de Circuitos, Barras e Fluxos de Potência dos Sistemas Testados 164

$\begin{array}{ll}\text { A1 Sistema de } 30 \text { Barras } & 164\end{array}$

$\begin{array}{ll}\text { A2 Sistema de } 39 \text { Barras } & 167\end{array}$

$\begin{array}{ll}\text { A3 Sistema de } 57 \text { Barras } & 171\end{array}$

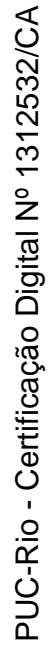

$\begin{array}{ll}\text { A4 Sistema de } 118 \text { Barras } & 176\end{array}$ 


\section{Lista de Figuras}

Figura 3.1 Modelo Equivalente m de Linha de Transmissão 34

Figura 3.2: Representação Geral dos Transformadores 35

Figura 3.3: Circuito Equivalente m de Transformador em Fase 37

Figura 3.4: Defasador Puro $(\mathrm{t}=\mathrm{e} j \boldsymbol{\varphi}) \quad 39$

Figura 3.5: Convenção do Sentido de Fluxo de Potência 42

Figura 3.6: Sequência de Passos FPC 52

Figura 4.1: Circuito de Duas Barras 58

Figura 4.2: Curvas no R3 de P1 como Função m 60

Figura 4.3: Curvas de V1 Constante no Plano ө1P1 61

Figura 4.4: P1 e Q1 Constantes no Plano 01V1 para Diferentes Valores de P1 e Q1 62

Figura 4.5: Soluções de Tensão na Carga com o Mesmo Fator de Potência 64

Figura 4.6: Curva para $\phi$ Constante no Plano S1V1 65

Figura 4.7: Circuito com as Impedâncias da Transmissão e da Carga 66

Figura 4.8: LET sobre as Curvas $\phi$ Constante no Plano SV 70

Figura 4.9: Sistema de Duas Barras com Capacitor na Barra Terminal $\quad 70$

Figura 4.10: Aumento e Diminuição da Tensão Devido à Introdução de um Capacitor $\quad 73$

Figura 4.11: $\phi$ e Zc Constantes no Plano SV com e sem Capacitor 74

Figura 4.12: Ângulo de Tensão Barra 1 (graus) 75

Figura 5.1:Localização dos Vetores Gradientes de Pi e Qi no Plano $\theta V$

Figura 5.2: Índices de Influência Positivo e Negativo 87

Figura 5.3: Barra de Tensão Controlada Operando nas Regiões Superior e Inferior da Curva para $\phi$ Constante no Plano SV 88

Figura 5.4: Variação da Tensão no Tempo em Barras de Tensão Controlada 89

Figura 5.5: Matriz Jacobiano Modificada para Análise de Barras de Tensão Controlada

Figura 5.6: Matriz Jacobiano Modificada para Análise de

Barras de Controle de Tensão Remoto 
Figura 5.7: Matriz Jacobiano Modificada para Análise de Barras $\theta \mathrm{V}$

Figura 5.8: Mariz Jacobiano Modificada para Análise de Barras de Tensão Controlada por Controle Coordenado de Tensão

Figura 5.9: Matriz Jacobiano Modificada para Análise de Barras de Controle Pertencentes ao Controle Coordenado de Tensão

Figura 5.10: Sistema de um Gerador e uma Carga Simples. 98

Figura 6.1: FPC de Todos os Sistemas Testados 107

Figura 6.2: Diagrama Unifilar do Sistema de 6 Barras 109

Figura 6.3: FPC Aplicado ao Sistema de 6 Barras 111

Figura 6.4: Diagrama Unifilar do Sistema de 14 Barras 116

Figura 6.5: FPC Aplicado ao Sistema de 14 Barras 119

Figura 6.6: Diagrama unifilar do Sistema de 30 Barras 123

Figura 6.7: FPC Aplicado ao Sistema de 30 Barras 124

Figura 6.8: Diagrama Unifilar do Sistema de 39 Barras 128

Figura 6.9: FPC Aplicado ao Sistema de 39 Barras 129

Figura 6.10: Diagrama Unifilar do Sistema de 57 Barras 135

Figura 6.11: FPC Aplicado ao Sistema de 57 Barras 136

Figura 6.12: Diagrama Unifilar do Sistema de 118 Barras 144

Figura 6.13: FPC Aplicado ao Sistema de 118 Barras 145 


\section{Lista de Tabelas}

Tabela 2.1: Tensões entre Fases Admissíveis a $60 \mathrm{~Hz}$.

Tabela 6.1: Dados de Rede do Sistema de 6 Barras 109

Tabela 6.2: Caso-Base do Sistema de 6 Barras 110

Tabela 6.3: Fluxos de Potência nos Ramos da Rede 110

Tabela 6.4: IETs do Caso-Base do Sistema de 6 Barras 111

Tabela 6.5: IETs do Sistema de 6 Barras a $60 \%$ do Carregamento Máximo

Tabela 6.6: IETs do Sistema de 6 Barras a $80 \%$ do Carregamento Máximo

Tabela 6.7: IETs do Sistema de 6 Barras a 90\% do Carregamento Máximo

Tabela 6.8: IETs do Sistema de 6 Barras a 95\% do Carregamento Máximo

Tabela 6.9: IETs do Sistema de 6 Barras a 97\% do Carregamento Máximo

Tabela 6.10: IETs do Sistema de 6 Barras a $98 \%$ do Carregamento Máximo

Tabela 6.11: IETs do Sistema de 6 Barras a 100\% do Carregamento Máximo

Tabela 6.12: Dados de Rede do Sistema 14 Barras

Tabela 6.13: Caso-Base do Sistema de 14 Barras

Tabela 6.14: Fluxos de Potência nos Ramos da Rede

Tabela 6.15: IETs do Caso-Base do Sistema de 14 Barras

Tabela 6.16: IETs do Sistema de 14 Barras a $60 \%$ do Carregamento Máximo

Tabela 6.17: IETs do Sistema de 14 Barras a $80 \%$ do Carregamento Máximo

Tabela 6.18: IETs do Sistema de 14 Barras a 90\% do Carregamento Máximo

Tabela 6.19: IETs do Sistema de 14 Barras a 95\% do Carregamento Máximo 
Tabela 6.20: IETs do Sistema de 14 Barras a 97\% do Carregamento Máximo

Tabela 6.21: IETs do Sistema de 14 Barras a 98\% do Carregamento Máximo

Tabela 6.22: IETs do Sistema de 14 Barras a 100\% do Carregamento Máximo

Tabela 6.23: IETs do Caso-Base do Sistema de 30 Barras

Tabela 6.24: IETs do Sistema de 30 Barras a 60\% do Carregamento Máximo

Tabela 6.25: IETs do Sistema de 30 Barras a $80 \%$ do Carregamento Máximo

Tabela 6.26: IETs do Sistema de 30 Barras a $90 \%$ do Carregamento Máximo

Tabela 6.27: IETs do Sistema de 30 Barras a 95\% do Carregamento Máximo

Tabela 6.29: IETs do Sistema de 30 Barras a 98\% do Carregamento Máximo

Tabela 6.30: IETs do Sistema de 30 Barras a $100 \%$ do Carregamento Máximo

Tabela 6.31: IETs do Caso-Base do Sistema de 39 Barras

129

Tabela 6.32: IETs do Sistema de 39 Barras a $60 \%$ do Carregamento Máximo

Tabela 6.33: IETs do Sistema de 39 Barras a $80 \%$ do Carregamento Máximo

Tabela 6.34: IETs do Sistema de 39 Barras a 90\% do Carregamento Máximo

Tabela 6.35: IETs do Sistema de 39 Barras a 95\% do Carregamento Máximo

Tabela 6.36: IETs do Sistema de 39 Barras a 97\% do Carregamento Máximo

Tabela 6.37: IETs do Sistema de 39 Barras a 98\% do Carregamento Máximo

Tabela 6.38: IETs do Sistema de 39 Barras a 99\% do Carregamento Máximo 
Tabela 6.39: IETs do Sistema de 39 Barras a $100 \%$ do Carregamento Máximo

Tabela 6.40: IETs do Caso-Base do Sistema de 57 Barras

Tabela 6.41: IETs do Sistema de 57 Barras a $60 \%$ do Carregamento Máximo

Tabela 6.42: IETs do Sistema de 57 Barras a $80 \%$ do Carregamento Máximo

Tabela 6.43: IETs do Sistema de 57 Barras a $90 \%$ do Carregamento Máximo

Tabela 6.44: IETs do Sistema de 57 Barras a $95 \%$ do Carregamento Máximo

Tabela 6.45: IETs do Sistema de 57 Barras a $97 \%$ do Carregamento Máximo

Tabela 6.46: IETs do Sistema de 57 Barras a $98 \%$ do Carregamento Máximo

Tabela 6.48: IETs do Sistema de 57 Barras a 100\% do Carregamento Máximo

Tabela 6.49: IETs do Caso-Base do Sistema de 118 Barras

Tabela 6.50: IETs do Sistema de 118 Barras a $60 \%$ do Carregamento Máximo

Tabela 6.51: IETs do Sistema de 118 Barras a 80\% do Carregamento Máximo

Tabela 6.52: IETs do Sistema de 118 Barras a 90\% do Carregamento Máximo

Tabela 6.53: IETs do Sistema de 118 Barras a 95\% do Carregamento Máximo

Tabela 6.54: IETs do Sistema de 118 Barras a 97\% do Carregamento Máximo

Tabela 6.55: IETs do Sistema de 118 Barras a $98 \%$ do Carregamento Máximo

Tabela 6.56: IETs do Sistema de 118 Barras a 99\% do Carregamento Máximo

Tabela 6.57: IETs do Sistema de 118 Barras a $100 \%$ do Carregamento Máximo 
Tabela 6.58: IETs com Limites de Geração no Caso-Base do Sistema de 118 Barras

Tabela 6.59: IETs com Limites de Geração a $60 \%$ do

Máximo Carregamento do Sistema de 118 Barras

Tabela 6.60: IETs com Limites de Geração a $65 \%$ do

Máximo Carregamento do Sistema de 118 Barras

Tabela 6.61: IETs com Limites de Geração a 66\% do

Máximo Carregamento do Sistema de 118 Barras

Tabela 7.1: Comparação Métodos de Avaliação de Estabilidade de Tensão 159

Tabela A1: Dados de Rede do Sistema de 30 barras 164

Tabela A2: Caso-Base do Sistema de 30 Barras 165

Tabela A3: Fluxos de Potência nos Ramos da Rede 166

Tabela A4: Dados de Rede do Sistema de 39 Barras 167

Tabela A5: Caso-Base do Sistema de 39 Barras 168

Tabela A6: Fluxos de Potência nos Ramos da Rede 169

Tabela A7: Dados de Rede do Sistema de 57 Barras 171

Tabela A8: Caso-Base do Sistema de 57 Barras 173

Tabela A9: Fluxos de Potência nos Ramos da Rede 174

Tabela A10: Dados de Rede do Sistema de 118 Barras 176

Tabela A11: Caso-Base do Sistema de 118 Barras 181

Tabela A12: Fluxos de Potência nos Ramos da Rede 184 


\section{Lista de Abreviaturas e Siglas}

ANEEL: Agência Nacional de Energia Elétrica

CC: Corrente Continua

ERAC: Esquema Regional de Alívio de Carga

FACTS:Flexible AC Transmission System

FPC:Fluxo de Potência Continuado

I: Corrente

II: Índice de Influência

IL: Índice L

LEEA: Limite de Estabilidade Estática Angular

LET: Limite de Estabilidad de Tensao

MST: Margem de Segurança de Tensão

ONS: Operador Nacional do Sistema

P: Potência Ativa

PMC: Ponto de Máximo Carregamento

Q: Potência Reativa

SEP: Sistema Elétrico de Potência

SIN: Sistema Interligado Nacional

V: Módulo da Tensão

VT: Vetor Tangente

Y: Admitância

Z: Impedância

Zc: Impedância de Carga no Máximo Carregamento

Zt: Impedância na Linha de Transmissao

$\theta$ : Refêrencia Angular

$\lambda$ : Fator de Carregamento

$\phi$ : Fator de Potência 